\title{
Decision support system for mass dispensing of medications for infectious disease outbreaks and bioterrorist attacks
}

\author{
Eva K. Lee · Siddhartha Maheshwary • \\ Jacquelyn Mason • William Glisson
}

Published online: 13 October 2006

(C) Springer Science + Business Media, LLC 2006

\begin{abstract}
A simulation and decision support system, RealOpt ${ }^{\odot}$, for planning large-scale emergency dispensing clinics to respond to biological threats and infectious disease outbreaks is described. The system allows public health administrators to investigate clinic design and staffing scenarios quickly.

$\mathrm{RealOpt}^{\odot}$ incorporates efficient optimization technology seamlessly interfaced with a simulation module. The system's correctness and computational advantage are validated via comparisons against simulation runs of the same model developed on a commercial system. Simulation studies to explore facility layout and staffing scenarios for smallpox vaccination and for an actual anthrax-treatment dispensing exercise and post event analysis are presented.

The system produces results consistent with the model built on the commercial system, but requires only a fraction of the computational time. Each smallpox scenario runs within 1 CPU minute on $\mathrm{RealOpt}^{\odot}$, versus run times of over 5-10 hours on the commercial system. The system's fast computational time enables its use in large-scale studies, in particular an anthrax response planning exercise involving a county with 864,000 households. The computational effort required for this exercise was roughly $30 \mathrm{~min}$ for all scenarios considered,
\end{abstract}

Preliminary results of this study were presented at INFORMS Colorado Oct. 2004. The system developed has been reported in the Department of Homeland Security IAIP Directorate Daily Open Source Infrastructure Report, "New Computer Program helps Health Departments Halt Outbreaks," Sept. 2004; and Bioscience Innovations, "Emergency treatment response for bioterrorism and infectious disease outbreak," Oct. 2004.

Eva K. Lee $(\bowtie) \cdot S$. Maheshwary

School of Industrial and Systems Engineering, Georgia

Institute of Technology, Atlanta, Georgia

e-mail: evakylee@isye.gatech.edu

Eva K. Lee

Winship Cancer Institute, Emory University School of Medicine, Atlanta, Georgia

J. Mason

Public Health Environmental Readiness Branch, Centers for Disease Control and Prevention

W. Glisson

DeKalb County Board of Health, Office of Emergency Preparedness 
demonstrating that $\mathrm{RealOpt}^{\odot}$ offers a very promising avenue for pursuing a comprehensive investigation involving a more diverse set of scenarios, and justifying work towards development of a robust system that can be widely deployed for use by state, local, and tribal health practitioners.

Using our staff allocation and assignments for the Anthrax field exercise, DeKalb county achieved the highest throughput among all counties that simultaneously conducted the same scale of Anthrax exercise at various locations, with labor usage at or below the other counties. Indeed, DeKalb exceeded the targeted number of households, and it processed 50\% more individuals compared to the second place county. None of the other counties achieved the targeted number of households. The external evaluators commented that DeKalb produced the most efficient floor plan (with no path crossing), the most cost-effective dispensing (lowest labor/throughput value), and the smoothest operations (shortest average wait time, average queue length, equalized utilization rate). The study proves that even without historical data, using our system one can plan ahead and be able to wisely estimate the required labor resources.

The exercise also revealed many areas that need attention during the operations planning and design of dispensing centers. The type of disaster being confronted (e.g., biological attack, infectious disease outbreak, or a natural disaster) also dictates different design considerations with respect to the dispensing clinic, facility locations, dispensing and backup strategies, and level of security protection. Depending on the situation, backup plans will be different, and the level of security and military personnel, as well as the number of healthcare workers required, will vary.

In summary, the study shows that a real-time decision support system is viable through careful design of a stand-alone simulator coupled with powerful tailor-designed optimization solvers. The flexibility of performing empirical tests quickly means the system is amenable for use in training and preparation, and for strategic planning before and during an emergency situation. The system facilitates analysis of "what-if" scenarios, and serves as an invaluable tool for operational planning and dynamic on-the-fly reconfigurations of large-scale emergency dispensing clinics. It also allows for "virtual field exercises" to be performed on the decision support system, offering insight into operations flow and bottlenecks when mass dispensing is required for a region with a large population. The system, designed in modular form with a flexible implementation, enables future expansion and modification regarding emergency center design with respect to treatment for different biological threats or disease outbreaks. Working with emergency response departments, further fine-tuning and development of the system will be made to address different biological attacks and infectious disease outbreaks, and to ensure its practicality and usability.

Keywords Bioterrorism - Infectious disease - Decision support system - Simulation · Optimization $\cdot$ Anthrax $\cdot$ Smallpox $\cdot$ Emergency response $\cdot$ Resource allocation

\section{Introduction}

Over the past decade, terrorists have employed varied tactics (see Alibek, 1999; Bellamy and Freedman, 2001; Scott and Duncan, 2001). These include dissemination of anthrax spores, intentional food product contamination, release of chemical weapons in major metropolitan subway systems, and suicide attacks using explosive devices and passenger airplanes. While all these acts can cause major panic and disruption, especially pernicious are the attacks that 
employ biological or chemical agents. Unfortunately, it is very difficult to accurately predict the time and place of such attacks. Therefore, a high degree of response preparedness is required.

Considerable research has been done in the area of bioterror emergency response and preparedness (see Franz et al., 1997; Kaufmann, Meltzer, and Schmid, 1997; Eubank, 2002; Hupert et al., 2003). Many authors have studied general ideas on being prepared for a bioterror attack and how to respond to it. Okumura et al. (1998) study the Tokyo subway sarin attack. They provide the details related to how the community emergency medical services system responded from the viewpoint of disaster management, the problems encountered, and how they were addressed. Keim and Kaufman (1999) study and discuss the need to come up with a systematic structure and recommendations that emergency response officials can utilize efficiently. Noji (2003) outlines steps that have been taken in the USA for strengthening medical and public health capacity to protect against these dangers. Along similar lines, Sidel, Gould, and Cohen (2002) discuss the issue that preparing for bioterror is increasingly being interpreted from the viewpoint of the military, and not enough attention is being paid to the preparedness of the public health infrastructure. Rotz and Hughes (2004) examine some of the recent advances in detecting and responding to threats from bioterrorism and emerging infectious disease and identify priorities for the future. Barnett et al. (2005) discuss the application of the Haddon matrix - a conceptual model used for more than two decades in injury prevention and response strategies-for the purposes of sound public health readiness and response capabilities. Other researchers have explored the application of surveillence and information technologies for enhancing preparedness against bioterror attacks (see Bravata et al., 2004; Nguyen, Rosen, and Koop, 2005). Another interesting area of research is the human aspect of bioterror preparedness and response (see Drenkard et al., 2002; Clizbe, 2004; Larkin and Arnold, 2004). Many researchers have tried to apply the tools of mathematical modeling to the area of bioterrorism. Larson (2004) reviews the major operations research related work done to date in emergency response. Many researchers have done work in modeling a smallpox attack (see Gani and Leach, 2001; Meltzer et al., 2001; Epstein et al., 2002; Fauci, 2002; Kaplan, Craft, and Wein, 2002, 2003; Kaplan, 2004). Other related works in this area include (Fast, O'Steen, and Addis, 1995; Centers for Disease Control, 2002; Reis and Mandl, 2003; Wein, Craft, and Kaplan, 2003).

In this paper, we concentrate on the scenario where a major city is hit by a bioterror attack. The response requires massive mobilization of the public health infrastructure. In particular, the administrative agencies need to setup many clinical facilities where mass population can come in for treatment. Planning for fast, efficient and smooth setup and operation of such facilities requires a powerful and flexible Decision Support System (DSS). The development and testing of such a tool is the main focus of this paper.

Considerable research has been spawned in the area of logistics security in the aftermath of the September 11 attacks. Although recent anthrax events have prompted some investigation of optimal staffing arrangements necessary for treatment centers, the study and sophistication of suitable model designs is still in its infancy. Hupert, Mushlin, and Callahan (2002) investigated the optimal staffing arrangements necessary to attain a specified patient throughput rate with no bottlenecks. In their study, they did not consider patient orientation and other issues related to station flows in the treatment center. Recently, Mason and Washington (see Centers for Disease Control, 2002 November; Mason and Washington, 2003) investigated optimal staffing arrangements in the face of limited staffing availability. The simulation system they developed, Maxi-Vac, included a patient orientation station and other stations, as described in the Centers for Disease Control reports (see Mason and Washington, 2003; Centers for Disease Control, 2002 August). The CDC study has proven to be 
invaluable, as it offers insight on the practicality of a simulation system in emergency situations. However, the implementation revealed bottlenecks between the commercial simulation software and the optimization software. Over ten hours are needed to obtain a usable feasible solution in each scenario. The developers of Maxi-Vac therefore solved their model with a selected set of inputs, and created look-up tables so that emergency health administrators can obtain solutions quickly. This limits the usefulness of the system, since users cannot enter inputs specific to their particular operating conditions (e.g. number and type of personnel available to staff the clinic); rather, they must choose from the selected pre-determined inputs. A subsequent field study by Giovachino and McCue (2003) provided crucial insights on the feasibility of the model developed and key issues that need to be addressed for practical use. Furthermore, it highlights the importance of a real-time system in which users can dynamically make changes in the floor plan in response to patient flow. These preliminary findings motivate our study herein of the development and implementation of a decision support system that can aid in large-scale, real-time resource allocation, while allowing users the flexibility to enter input values of their choosing. Such system will enable public health agencies to obtain results that better reflect their actual operating environment for preparation of emergency response.

\section{Problem description and model formulation}

\subsection{Large-scale dispensing centers for emergency response}

CDC and public health administrators work closely with one another to prepare for and document the steps required to administer medication in the event that mass dispensing is needed. The goal and objectives of a dispensing center are to deliver appropriate emergency services (e.g. vaccine, medical service, and education/training) to high-risk populations in an orderly, expeditious and safe manner. Within the center, the tasks and objectives include

- Assess health status of clients

- Assess eligibility of clients to receive service

- Assess implications of each case and refer case for further investigation if necessary

- Counsel clients regarding service and associated risks

- Administer service

- Educate regarding adverse events

- Document services

- Monitor vaccine take rates

- Monitor adverse reactions

- Monitor development of disease

In this section, a template for setting up a dispensing center is described. Depending on the nature of dispensing and disease, the dispensing process can be collapsed or expanded as necessary to decrease or increase the flow of clients through the center. Typically, multiple dispensing centers will be needed to accommodate the population in a given county. Countywide planning and coordination is therefore needed.

Areas must be set aside for eight primary activities in the dispensing process: (1) Assembly / Intake, (2) Triage, (3) Orientation, (4) Registration, (5) Screening, (6) Service, (7) Education and (8) Discharge. Below is a brief description of the tasks carried out at each of these activity areas.

且Springer 
1. Assembly/Intake - County residents (clients) will be instructed by public announcements to assemble at a specified location (or possibly multiple locations). From the assembly area(s), clients will be shuttled to a Point-of-Dispensing (POD). They will be given a medical history form to fill out while being transported to the POD and while waiting in the POD intake lines. POD Intake Greeters will check the medical history forms for legibility, correctness and completeness while the clients are waiting in the intake line. If the lines are long, general information sheets will be distributed to those in line. Individual clients who are identified as obviously ill will be isolated and transported to a medical treatment center. The remaining clients will be directed to the triage area. Security personnel will be posted at both the assembly areas and the PODs to manage crowd control.

2. Triage-Nurses, Emergency Medical Services personnel, Physicians, Physician Assistants, and Mental Health Counselors will man the Triage area. Clients will be separated into three groups as they are assessed by Triage staff: sick clients, the worried but well, and apparently healthy clients. The first group of clients - those with any apparent illnesswill be immediately directed to the Sick Area for further evaluation and/or medical assistance. An Epidemiology Liaison located at the POD will be notified if there is a potential case of the disease. If the client is deemed critical, the medical staff will stabilize the individual and transport him/her via Emergency Medical Services to a medical facility (e.g. hospital), or as directed. The second group of distressed clients are those who are apparently physically healthy but are overwhelmed or experiencing a high degree of anxiety. These persons, the "worried well", will be directed to Mental Health Services for further assistance, since their anxiety may interfere with their services or services provided to others in the POD. The third group of clients - those with no obvious symptoms-will be directed to the Greeter for Orientation.

For a contagious disease, the triage process will separate the clients into those with symptomatic disease (and thus not eligible for vaccine), those exposed to disease and yet still capable of benefiting from vaccination, and those not yet exposed. The latter two groups are candidates for vaccination; the first group is taken elsewhere for treatment for the disease, and the middle group is identified so that contact tracing can take place.

3. Orientation-Educators will run concurrent sessions, possibly with the aid of educational videos, for groups of approximately $25-30$ clients. The group size may be adjusted depending on staff availability and clients' level of understanding. General information will be presented regarding the situation, prophylaxis and/or treatment, common adverse affects, and instructions for completing the regimen. Informational materials will be handed out to reinforce this information. Orientation also is an opportunity for clients to start filling out any additional required forms. Orientation staff will provide instruction and assistance in completing these forms.

Clients with certain conditions or special needs that require private counseling will be provided the appropriate prophylaxis and then referred to their private physician for follow-up and in-depth consultation regarding drug, or drug-disease interactions. Upon completing orientation, clients will be directed to the registration area.

4. Registration-At the registration area, clients will sign in on a numerically numbered sign-in log sheet, and will be given a corresponding numbered identification card. This number will be used to identify and track clients, and will serve as the prescription number. Clients will sign a Patient Consent Form, and complete any remaining forms. Staff will review forms for legibility, accuracy, and completeness; and assist clients who have difficulties in completing forms. Clients will be instructed to keep the forms with them for presentation at the remaining stations, and for eventual collection at the exit station. 
5. Screening-Screeners will review the medical forms and interview the client. With the client, the screeners will review information about the disease or problem, and discuss the services that would be appropriate for the individual client, including the benefits and risks of services. The screeners will reinforce the "Fact Sheet" and "Frequently Asked Question" sheet; provide uniform information, exposure risk assessment, purpose and duration of prophylaxis, side effects, effects of refusal of prophylaxis, and the medication distribution process. The screeners will also provide information on medications to be dispensed and the reasons for various forms of prophylaxis (i.e. pregnancy, children, medication interactions, etc.).

Clients with possible contraindications, complex health problems or more complex problems will be referred to Senior Screeners, who are responsible for managing those persons with more complex issues. The senior screeners will review information about the disease, the risks and benefits of the service, and specific recommendations with individual clients.

At this juncture, clients in both groups may elect to receive service or not. Clients who opt to receive service will be directed to the Service Area and subsequently to the Education Area. Clients who decide not to receive service will be directed to the Education Area.

6. Dispensing service area-A pharmacist will be present to oversee the dispensing operation. All dispensing will be recorded. After receiving service, clients will be directed to the Education Area.

7. Education area-Educators will reinforce key messages about the disease and ensure that clients are aware of relevant issues regarding service. Educators should be aware of all potential adverse reactions to the medication or immunization being dispensed, and should be vigilent in identifying clients with such reactions. If a client has an adverse reaction, (s)he will be swiftly moved to the Sick Area. In the Sick Area, staff will assess the problem, stabilize the condition and make a decision to transport the person by the EMS vehicle to an appropriate medical care facility. Clients with no adverse reaction will be directed to Discharge, where forms will be collected and staff will ensure that clients' needs are fulfilled prior to release.

8. Discharge area-Staff will review all documentation for completion and legibility. All forms will be placed in locked data information holders for retrieval by Logistics runners. Signs will be posted to direct clients to the parking area to board the transportation vehicles for the return trip to the assembly sites.

During any phase of the client flow, clients who are overwhelmed or experience anxiety can be referred to Mental Health Services in the POD. This is critical as client anxiety in a densely-crowded POD could trigger group disruption that could be detrimental to the dispensing service as well as security within the POD.

It should be remarked that there exists similarity between POD design and manufacturing and service systems design, and that there exists a large body of literature on the latter. Decision variables in the context of manufacturing systems may pertain to resource allocation, buffer allocation or other decisions influencing system performance. Queuing theory, simulation, and optimization are the primary technologies used to address design problems (see Hopp and Spearman, 2000; Buzacott and Shanthikumar, 1993; Papadopoulos, Heavey, and Browne, 1993; Viswanadham and Narahari, 1992; Altiok, 1997; Eglese, 1990; Spinellis and Papadopoulos, 2000a; Tompkins and Azadivar, 1995; Spinellis and Papadopoulos, 2000b; Heavey, Papadopoulos, and Browne, 1993; Gershwin, 1987; Dallery and Frein, 1993; Law and Kelton, 1991; Laguna and Marti, 2003).

There are, however, key differences between manufacturing and service system design problems and POD design problems. Chief among them are the number of stochastic factors 
involved and the difficulty in obtaining a priori estimates of parameters and distributions. The human element and the unfamiliarity with the process in a large-scale emergency situation (by both the patients receiving treatment and the health care workers administering it) increase the level of design complexity tremendously. This complexity is vividly illustrated and reported in the Arlington field study (see Giovachino and McCue, 2003). Furthermore, the field test concludes and emphasizes that the key to success is flexibility, both of staff to be able to perform a number of different duties and of facility to be readily reconfigured on-the-fly. The only feasible strategy is to set up a clinic based on the best estimates available, and then be prepared to reconfigure it while it operates. Clearly, a decision support system (DSS) that facilitates analysis of "what-if" scenarios will be an invaluable tool for planning such reconfigurations.

\subsection{Resource allocation model and formulation}

The POD facility can be modeled as an open queuing network comprised of a number of stations that form the nodes of a directed graph. (See Gross and Harris (1998) for a discussion of open queuing networks.) Patients arrive at the center according to some stochastic process. At each station, multiple servers may be present. Types of servers include nurses, medical technologists, mental health counselors, physicians, physician assistants, pharmacists and volunteers, depending on the expertise required by the service being rendered. The various service times follow some probability distributions.

The layout of the stations within a POD can be done with the aid of a specialized graph drawing tool. Once a layout is selected, the assignment of workers to stations can be formulated as an optimization problem as follows.

Let $S=\{1, \ldots, s\}$ be the set of work-stations, and $T=\{1, \ldots, t\}$ be the set of workertypes that are available. We define integer decision variable $x_{i j} \in Z_{+}$as the number of workers of type $i \in T$ assigned to station $j \in S$. Let $k_{i j}$ be the cost of assigning a worker of type $i$ to station $j, i \in T, j \in S$. Let $\bar{m}_{i j}$ and $\underline{m}_{i j}$ be, respectively, the maximum and minimum number of workers of type $i$ that may be assigned to station $j$, for $i \in T, j \in S$.

Let $w_{j}, q_{j}$ and $u_{j}$ be the average wait time, average queue length and average utilization rate, respectively, at station $j, j \in S$. Let $c$ be the average cycle time (length of time a patient spends in the system), and let $\theta$ be the average throughput (number of patients serviced in a specified time period). The cost at each station $j$ can be represented as $g_{j}\left(\sum_{i \in T} k_{i j} x_{i j}, w_{j}, q_{j}, u_{j}\right), j \in S$. The total system cost depends on the cost at each station, and also on system parameters such as cycle time and throughput. Thus, the total cost may be represented as $f\left(\sum_{j \in S} g_{j}, c, \theta\right)$. Here, $g_{j}$ and $f$ are functions that are not necessarily expressible in closed form. A general representation of the resource allocation problem can now be formulated as

$$
\begin{aligned}
& \min z=f\left(\sum_{j \in S} g_{j}, c, \theta\right) \\
& \text { s.t. } \quad \underline{m}_{i j} \leq x_{i j} \leq \bar{m}_{i j} \quad \forall i \in T, j \in S \\
& \sum_{i \in K} \sum_{j \in S} x_{i j} \leq n_{K} \quad \forall K \in \bar{T} \\
& x_{i j} \in \mathbb{Z}_{+} \quad \forall i \in T, j \in S
\end{aligned}
$$


Here, $\bar{T}$ denotes a collection of subsets of $T$, and for $K \in \bar{T}, n_{K}$ is the number of available workers who can take on the role of the worker types represented by $K$.

The first set of constraints, Eq. (1), stipulates lower and upper limits on the number of workers of a given type that may be allocated to a given station. The second set of constraints, Eq. (2), is general and subsumes most restrictions that may be placed on the availability of workers. Finally, the last set of constraints, Eq. (3), imposes integrality on the decision variables. Equations (1)-(3) form the basis of the resource allocation problem. Other side constraints include constraining the average cycle time to be less than a pre-specified upper bound. This is critical for emergency response, since individuals should be moved through the system as quickly as possible. Our CDC collaborator has indicated that an acceptable upper bound is $150 \mathrm{~min}$.

This is a combinatorial optimization or mixed integer programming problem (MIP) with a non-linear (multiple) objective function. To be of use in a real emergency medical facility, one cannot place a priori restrictions on the type of probability distributions that are used to model arrival processes and service times. Indeed, as alluded to in the Arlington field tests report, a practical system needs to be flexible in allowing for changes in probability distribution for different regional usage. Under such general conditions, the objective function does not have a closed-form (see Gross and Harris, 1998). Therefore, conventional theoretical analysis via mathematical programming techniques are not applicable here.

The existing queuing research relies on several assumptions in order to analyze such models (see Buzacott and Shanthikumar, 1993; Gross and Harris, 1998). Most common approaches assume that the arrival process is Poisson and that the service times are exponentially distributed. In this case, the queuing network can be analyzed as a continuous-time Markov Chain (see Ross, 1995). Then, one may get closed form expressions for many of the system parameters, assuming the steady state has been reached. However, such analysis is not of much practicality. First, the assumptions on arrival processes and service time distributions are highly restrictive, and seriously compromise the realism of the system. Second, the dispensing facility might not operate in a steady state since we are dealing with large-scale emergencies. Finally, there is an element of unquantifiable uncertainty because we are dealing with human beings working and receiving treatments in extremely difficult conditions psychologically.

In this paper, our work is motivated strongly by CDC preliminary studies, by the Arlington field tests, and by our experience in an acutal anthrax emergency response exercise. We focus on developing efficient computational models, and present results based on the design of a computational decision support system RealOpt ${ }^{\odot}$ (Lee and Maheshwary, 2004; RealOpt User Manual, 2004-2006) that we have implemented.

\subsection{Computational approach}

To address the complexity of the problem and to allow flexibility in testing numerous scenarios and the dynamic resource allocation capability, we have designed and implemented a standalone, large-scale computerized decision support system, RealOpt ${ }^{\odot}$. There are three core components in the system: The Simulation Manager, The Optimization Manager, and The User Interface Manager and Linker Module. The Simulation Manager is responsible for running the simulations and extracting the various statistics (e.g., average wait-time, average queue length, average utilization rate, etc.) out of the treatment center. The Optimization Manager contains the various exact algorithms and fast heuristics and calls the Simulation Manager repeatedly to resolve and update resource allocation statistics. Finally the User 
Interface Manager is responsible for all the functions related to input of data and displaying of results. It includes a graph-drawing algorithm that allows users to design specific floor plans pertinent to the available facility and type of treatment (e.g., for smallpox, pandemic flu, anthrax). These three components integrate seamlessly within the decision support system.

Below we briefly explain the design of the three components. The entire system is developed using Java for easy portability across different computer platforms and personal digital assistants (PDAs).

I. The simulation manager. is a complex software module that allows flexibility in input parameters to facilitate investigating behavior and bottlenecks in the system under different scenarios. Figure 1 shows the organizational chart within our Simulation Manager.

The sub-module Blocks encapsulates the information related to various stages encountered by the incoming patients. These stages are represented by various blocks as shown in the figure. Each of these blocks has a specific function. For example, Process Blocks represent those stages where patients are served by a healthcare personnel. Thus, they represent manned stages. Similarly, Decision Blocks represent those stages where incoming patients are directed along divergent paths, to different blocks further down the flow. Note that in our simulation model for a medical facility, there are several blocks of each type to describe the real situation. The diagram here is simplied for easy explanation. Links, like Blocks, is also a structure element, and it encapsulates the information related to the connection from one block to another. Thus, Links contain the information about the various possible paths patients might take through the system. The sub-module Probability Distribution Manger encapsulates information about various probability distributions used by the sub-module Random Variate Manager, which is responsible for generating the random numbers used in the simulation. Simulation Model Info is a component that contains some basic information related to the simulation, such as terminating conditions, number of replications performed in each simulation, etc. Finally, Statistics Manager is responsible for generating the various statistics such as utilization, average queue lengths, average wait times, etc., at various Process Blocks.

The model assumes that the service times at each station are independent. In other words, if a person takes a long time at one station this does not make it more likely that he/she will take a long time at other stations. The empirical data in the field study supports this assumption except in the case of non-English speakers. It also assumes that all family members move together through the dispensing center (this is reasonable in the case of emergency response situations); all family members have the same level of potential exposure; and a time function

\section{Simulation Manager}
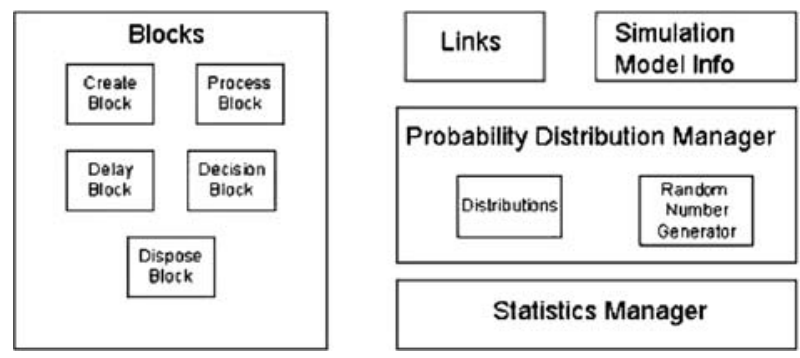

Fig. 1 A simplified Simulation Manager organizational chart. Note that in our actual simulation environment for a dispensing facility, there are several blocks of each type to describe the real situation. The diagram here is simplified for easy viewing 
(e.g., piecewise linear, quadratic) exists between mean provider service time and the number of people in a family.

II. The optimization manager. carries the responsibility of applying the various exact algorithms and fast heuristics to optimize the system. It does so by repeatedly calling the Simulation Manager, and based on the statistics generated by the Statistics Manager, decides how best to modify the system in order to improve the objective function(s). The optimization manager can accommodate various and multiple objectives. Possible objectives include maximize throughput, minimize staff usage to satisfy a specified throughput, minimize the maximum cycle time, minimize the maximum wait time/queue length, equalize the utilization rate at each station, etc. For the computational work reported in Sections 2.1 and 2.2, we maximize throughput and minimize average cycle time; as well as given a pre-set throughput, we minimize required resources, minimize average cycle time, and equalize the utilization rates. These objective function combinations, as well as other combinations, can be easily selected via the User-interface Manager.

The complexity of the problem and the need for real-time dynamic changes demand that a very fast heuristic procedure be in place. Since the performance of any heuristic algorithm depends on its implementation and on the structure/type of problem being solved, our heuristic algorithmic design is strongly guided by the real problem instances we face with mass dispensing of smallpox and anthrax prophylactics. For our own analysis, an exact optimization algorithm is also implemented to enable the measurement of tradeoffs between optimal solutions versus near-optimal (heuristic) solutions, and hence a heuristic's suitablity in the decision support system.

For this paper, we utilize a hybrid heuristic algorithm that is a cross between a greedy algorithm and a local search. In this heuristic, the first stage is used for initialization of the system. It works by greedily assigning workers to various stations within the center. The assignment is based on initial estimates on queue length, which are in turn based on the existing probability density functions at each station. Once all the workers have been assigned this way, the second stage takes over. The algorithm observes and registers the formation of queues with various lengths at different stations, and quickly and dynamically performs local search to swap local workers between various stations in an attempt to improve the objective value. The implementation of this algorithm is guided strongly by the layout design, worker types, and links between different work-stations within the emergency center.

This heuristic routine is ad-hoc, and function evaluation is performed via simulation, and thus can be computationally very expensive. Local search alone has been shown to be very efficient for large-scale network flow problems, and greedy algorithms alone can be very efficient for some classes of combinatorial problems. Our heuristic algorithm is designed specifically for resource allocation and has been tested and shown to offer a fast and robust solution engine within the simulation process. Figure 2 illustrates the integration of the Simulation Manager and the Optimization Manager within the decision support system.

III. The user interface manager and linker module. provides facilities for users to (a) design and review the floor plan and make modifications if necessary; (b) input data for starting a simulation study; and (c) review and extract output statistics to identify flow patterns within the system.

The design and reconfiguration of the dispensing facility is rendered by the end-user with the aid of a Graph Drawing Tool. The drawing tool provides a drag-and-drop interface for laying out various stations of the dispensing facility and linking them with arrows. The drawing tool is coupled with the Data Input Module which allows a user to enter alphanumeric 


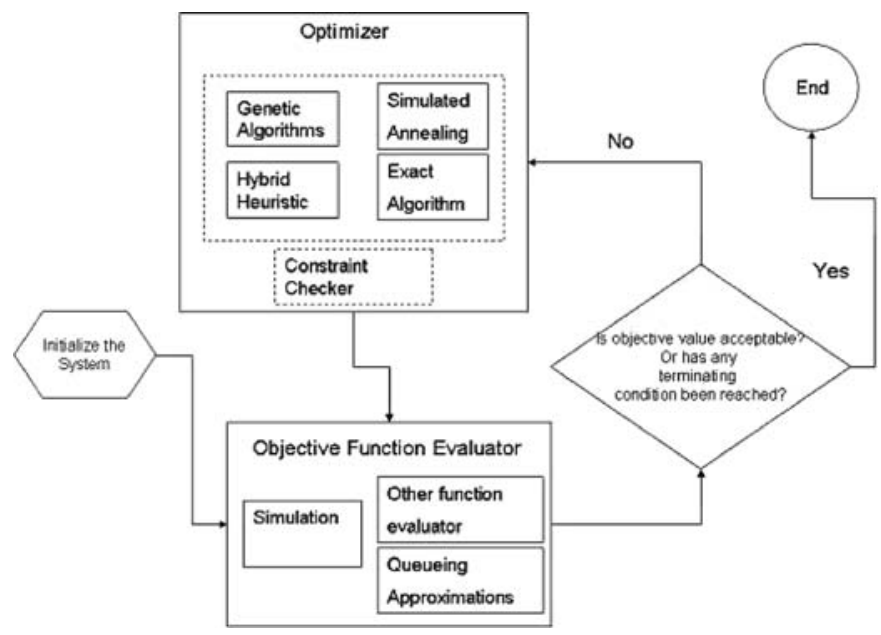

Fig. 2 Integration of the Simulation Manager and the Optimization Manager within the RealOpt ${ }^{\complement}$ decision support system

data such as (i) the name/description, service time distribution, and type of worker for each station; (ii) the availability of different types of healthcare personnel; (iii) the duration of the simulation period; (iv) desired objectives and constraints; and (v) the minimum level of expected throughput (in the case where minimizing staff resources is the chosen objective). A toolbar provides buttons for saving an existing configuration to a file and opening a previously saved configuration.

Additional design and implementation will be done in the future regarding customization for use by public health administrators.

The User Interface Manager is coupled with the Linker Module that, given a facility layout, automatically sets up the necessary framework for carrying out the simulation and optimization. Thus, the end-user of the system needs not have any specialized knowledge of simulation or optimization.

Upon completion of the simulation and optimization, the system returns solution statistics for the user. The statistics include the assignment of workers to each work-station, number of patients throughput over the simulation period, and detailed summary statistics for each work-station (e.g., number of patients served, average queue length, average utilization rate, average wait time, and worker-type allocation summary). Tables and graphs can be generated for quick review of patterns in patient flow within the system.

\section{Computational and field studies}

Given the flexibility that our system allows, one can generate an enormous amount of numerical data based on different input parameters. As an initial validation of our system, we replicated the smallpox tests performed by Mason and Washington (2003), and benchmarked our running times and results against simulation runs using a commercial system. We also participated in a recent study with a local county state health emergency division preparing for an actual anthrax-treatment dispensing exercise. Section 2.1 summarizes the small pox study, and Section 2.2 describes the planning and post-event analysis for the Anthrax field exercise. 


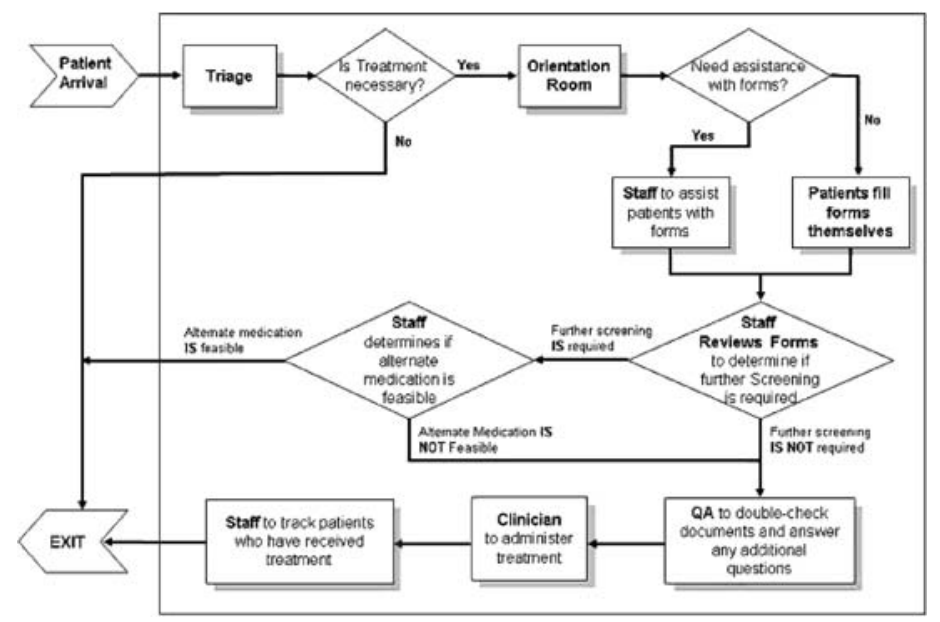

Fig. 3 A smallpox vaccination clinic design with flows along various stations

\subsection{Smallpox vaccination study}

Figure 3 shows an emergency vaccination center for smallpox, designed according to the guidelines described in Section 1.1, as documented in CDC reports (see Mason and Washington, 2003; Centers for Disease Control, 2002 August). Patients enter the clinic and are subjected to a triage process that separates them into those with symptomatic disease (and thus not eligible for vaccine), those exposed to disease and yet still capable of benefiting from vaccination, and those not yet exposed. The latter two groups are candidates for vaccination; the first group is taken elsewhere for treatment for the disease, and the middle group is identified so that contact tracing can take place. All patients except those with the disease then receive informational material and a form to fill out regarding their medical condition, and enter the clinic proper.

In the clinic, they enter one of several orientation rooms and see a videotape describing the disease, the vaccination, and medical conditions such as pregnancy that contraindicate vaccination. The videotape also alerts them to the fact that in some instances, the medical condition of a household member may contraindicate vaccination. Knowledgeable after having seen the video, they exit the video screening room and complete the medical forms they were given earlier. Reviewers screen these forms, separating those patients who can clearly be vaccinated from those in need of additional medical counseling, and perhaps even examination. The former group are sent to the second screening, QA, where staff will double check the forms and then send them straight to vaccination itself; the latter are sent to a further counseling and examination area, from which some are sent to QA for continuation to vaccination itself and others are directed to exit the facility without vaccination, since there is medical reason to believe that they ought not to be vaccinated. Those vaccinated then proceed to a final station at which they are informed how to care for themselves after vaccination (e.g., when to replace the bandage, and how long to keep wearing bandages), and are reminded how to protect others from contracting vaccinia from them.

Based on this clinic design and variations of it, we built a simulation model using a commercial simulation software package in which a commercial optimization system was integrated within the process. Immediately, bottlenecks of this system were observed, like 
those reported by Mason and Washington in the CDC study (Centers for Disease Control, 2002 November; Mason and Washington, 2003).

To gain insight into the cause of such bottlenecks, we designed a simulator from scratch, and coupled it with a specialized, fast heuristic optimization routine in a stand-alone system, as briefly described in Section 1.3. Our system RealOpt ${ }^{\odot}$ (Resource allocation Optimization) (Lee and Maheshwary, 2004; RealOpt User Manual, 2004-2006; Lee, Maheshwary, and Mason, 2005) was built in-house in Java to allow for portability across different computer platforms and PDAs. We used the system to gain better insight into the planning process, the tradeoffs and bottlenecks within the center setup, and the overall throughput performance; and to assess the computational capabilities of the system design.

As mentioned elsewhere, the CDC prototype system used a commercial simulator and a commercial optimizer, and each test required over ten hours of running time to arrive at a feasible solution. In contrast, identical tests run on $\mathrm{RealOpt}^{\odot}$ required less than one CPU minute.

We next used RealOpt ${ }^{\odot}$ to analyze the effect of variations in floor plans, probability distributions, interarrival times, simulation duration periods, and a range of different numbers pertaining to availability of healthcare workers. As part of this study, we observed the change in throughput, cycle time, average wait time, average queue length, and utilization of each station against the availability of workers. Given a specified inter-arrival rate of individuals to the treatment center, and specified service rates, the experiments were designed to uncover the point at which throughput performance and cycle time can no longer be improved by allocating additional staff. For example, we observe that given a certain distribution, an increase of workers from 15 to 17 improves the throughput performance over $8 \%$ and reduces cycle time by $50 \%$. In contrast, when increasing from 18 to 30 workers, throughput improves less than $1 \%$ whle the reduction in cycle time is about $18 \%$. This type of information is critical when preparing for mass vaccination of a regional population. Thus, the system developed can be an effective tool in planning how resources should be allocated, and estimating the levels of performance that can be achieved.

In addition to running simulations using parameters suggested by CDC studies, we also performed simulations using actual field measurements from the Arlington field study (Giovachino and McCue, 2003). Results of this study were reported in a paper to appear in Medical Decision Making (Lee, Maheshwary, and Mason, 2005).

\subsection{An anthrax field exercise-planning, exercise, and post-event analysis}

Working with the Office of Emergency Preparedness at the DeKalb County Board of Health (DeKalb County, Georgia, USA) and with CDC, we helped prepare for an anthrax emergency drill by performing simulations using $\mathrm{RealOpt}^{\odot}$ to determine the minimum staff resources needed to dispense anthrax prophylactic for a region.

The anthrax emergency drill involves the distribution of a medication for anthrax to the head of a household. In this case, broadcasting of the distribution of anthrax medication is first made through public announcements on television, radio and other news media, with clear information on the type of medication dispensed as well as forms that individual families should fill out as they go to their assigned emergency treatment clinic to pick up the medication. The regional planning involves the arrival of individuals (a representative for each household) at the Assembly Point where each person goes through the security checkpoint, and each will be given an opportunity to receive medical counseling 
before being bused to the point-of-dispensing (POD) to pick up medication for his/her entire family.

\subsubsection{Planning for the Anthrax exercise}

For the actual field exercise conducted in July 2005 in Atlanta, in addition to the office of emergency preparedness, the exercise involved the Fire, Police and School Departments.

The design of the Anthrax exercise called for separation of the Assembly Point and the Point-of-Dispensing. The reason for this separation is that in the event of a bioterror attack and subsequent emergency mass dispensing, one would need to secure the POD to prevent an attack on it, and do it with minimal disruption to the POD operation. Thus, a tight security check would be performed by law enforcement or military personnel at a separate site (the Assembly Point) and all cleared clients would then be bused to the POD.

The Assembly Point consists of three main blocks: Active Triage, Medical Evaluation, and Mental Health Evaluation. Active Triage implies that staff will actively greet and ask clients if they have questions. Clients first arrive at Active Triage from security. From Active Triage, $95 \%$ exit to be bused to POD, about $2 \%$ of the clients go to Medical Evaluation, $2 \%$ go to Mental Health Evaluation, and remaining $1 \%$ exit the system.

Upon entering medical evaluation for the first time, about 50 percent of the clients go to bus ride, 35 percent exit the system, and 15 percent go to mental health evaluation. Upon entering mental health evaluation for the first time, 50 percent go to bus ride, 35 percent exit the system, and 15 percent go to medical evaluation. When clients enter medical evaluation for the second time, 50 percent go to bus ride, and 50 percent exit the system. Similarly, when clients enter mental health evaluation for the second time, 50 percent go to bus ride, and 50 percent exit the system.

The POD consists of four main blocks: Passive Triage, Medical Evaluation, Mental Health Evaluation and Drug Dispensing. At Passive Triage, staff will be available, and they will wait for clients to direct specific enquiries to them. Clients first arrive at Triage. From Triage, about $2 \%$ of the clients go to Medical Evaluation, $2 \%$ go to Mental Health Evaluation, $95 \%$ go to Drug Dispensing, and the remaining $1 \%$ exit the system.

Upon entering Medical Evaluation for the first time, about $50 \%$ of the clients go to Drug Dispensing, 35\% exit the system, and $15 \%$ go to Mental Health Evaluation. Upon entering Mental Health Evaluation for the first time, $50 \%$ go to Drug Dispensing, 35\% exit the system, and $15 \%$ go to Medical Evaluation.

When clients enter Medical Evaluation for the second time, 50\% go to Drug Dispensing, and $50 \%$ exit the system. Similarly, when clients enter Mental Health Evaluation for the second time, $50 \%$ go to Drug Dispensing, and 50\% exit the system.

Figure 4 shows the flow through the Assembly Point and the POD.

Table 1 shows the service times estimated at each station for the Assembly Point and the POD, respectively, as estimated by the county emergency health officers. Working closely with the emergency response team, percentages and service times at each of the stations were estimated. We caution that these values are by no means historical, as there is no real data to rely on. Rather, these are purely estimated values to facilitate performing simulation scenarios to provide the minimal required staffing to man each station for the Anthrax exercise. The actual exercise in turn would provide us with some insight on service times for future emergency planning.

For the actual exercise, the Assembly Point was setup in an open parking lot of a mall, whereas the POD was setup in a high school. Buses were used to pick up clients at various locations, and transport them to the entrance of the Assembly Point. Buses were also em且Springer 


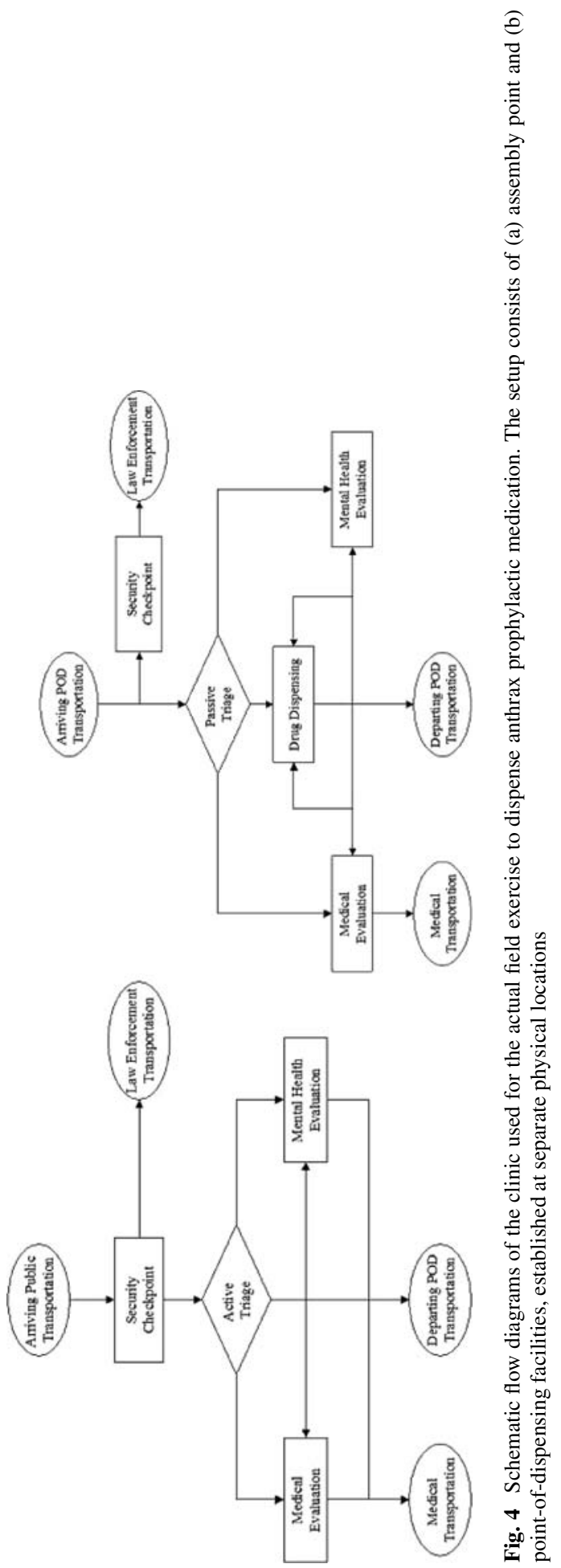


Table 1 Anthrax dispensing study

\begin{tabular}{lll}
\hline & Distribution & (min, mean, max) seconds \\
\hline Estimated parameters for assembly point & simulation \\
Triage & Triangular & $(30,60,120)$ \\
Med Eval & Triangular & $(300,450,600)$ \\
Mental Health Eval & Triangular & $(900,1050,1200)$ \\
Estimated parameters for point-of-dispensing simulation \\
Bus ride & Triangular & $(1080,1200,1800)$ \\
Triage & Triangular & $(1,10,60)$ \\
Drug Dispensing & Triangular & $(6,15,60)$ \\
Med Eval & Triangular & $(300,450,600)$ \\
Mental Health Eval & Triangular & $(900,1050,1200)$ \\
\hline
\end{tabular}

ployed to transport cleared clients from Assembly Point to the POD. The Georgia Institute of Technology team assisted in designing the actual layout of the POD in the high school to ensure optimal and smooth flow of clients.

The optimization objective for anthrax emergency drill is to determine the minimum number of workers needed at each station to achieve the required target throughput. The regional target population is 864,000 households. The county emergency planning unit has determined that 5 Assembly-Point and POD pairs should be used to get the medication dispensed within a 24-hour period. This in itself is a facility location problem, for which an integer program is used to determine the optimal facility locations and the number of facilities to be used. To avoid overcrowdedness, the county planned to even out the workload among all locations. In actual practice, it may be advantageous to have certain locations process more households than others, but that option was not explored in this exercise. Thus, for each Assembly-Point and POD pair, a minimum of 7,200 households must be processed within an hour. As such, within a 12 hours shift, we must process 86,400 households for each Assembly Point-POD pair. The tests were run for five target through puts: $20 \%, 40 \%, 60 \%$, $80 \%$, and $100 \%$ of the total targeted households. Thus, there were 5 scenarios for Assembly Point, and for POD. Each run was for 12 hours of simulated time, reflecting a 12-hour shift for each worker.

We ran simulations varying the dispensing time. The results indicate that the service time at drug dispensing has a significant impact on total worker allocation.

Figure 5 and Table 2 show the resource allocations for dispensing to 20\%, 40\%, 60\%, $80 \%$ and $100 \%$ of the targeted households for each Assembly Point and POD for a 12-hour shift. As the target level increases, labor increases, however, at a rate which is below a linear

Table 2 Staff allocation with the assumed estimated distributions

\begin{tabular}{|c|c|c|c|c|c|c|c|c|c|c|}
\hline \multirow[b]{2}{*}{$\begin{array}{l}\% \\
\text { Household }\end{array}$} & \multicolumn{4}{|c|}{ Assembly point } & \multicolumn{5}{|c|}{ Point-of-dispensing, POD } & \multirow[b]{2}{*}{ Total staff } \\
\hline & $\begin{array}{l}\text { Active } \\
\text { triage }\end{array}$ & Medical & $\begin{array}{l}\text { Mental } \\
\text { health }\end{array}$ & $\begin{array}{l}\text { Total } \\
\text { staff }\end{array}$ & $\begin{array}{l}\text { Passive } \\
\text { triage }\end{array}$ & Medical & $\begin{array}{l}\text { Mental } \\
\text { health }\end{array}$ & $\begin{array}{l}\text { Drug } \\
\text { dispensing }\end{array}$ & $\begin{array}{l}\text { Total } \\
\text { staff }\end{array}$ & \\
\hline 20 & 44 & 4 & 8 & 56 & 15 & 3 & 4 & 21 & 43 & 99 \\
\hline 40 & 87 & 7 & 14 & 108 & 29 & 4 & 7 & 42 & 82 & 190 \\
\hline 60 & 131 & 10 & 22 & 163 & 43 & 5 & 10 & 62 & 120 & 283 \\
\hline 80 & 132 & 13 & 27 & 172 & 44 & 7 & 15 & 64 & 130 & 302 \\
\hline 100 & 176 & 16 & 34 & 226 & 58 & 9 & 18 & 85 & 170 & 396 \\
\hline
\end{tabular}



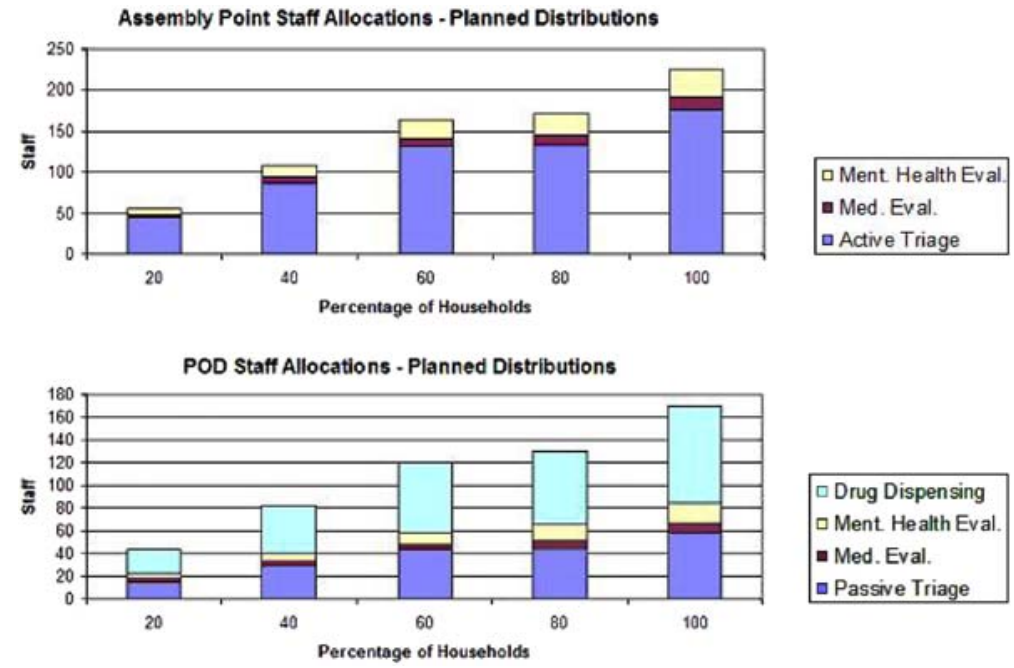

Fig. 5 Resource allocation with planned distributions for a 12-hour shift for a single Assembly Point and POD

correlation. Specifically, we observe that as the percentage of household increases, especially beyond $60 \%$, the use of labor becomes more efficient.

In the final preparedness for the Anthrax exercise, the results from the simulation study were used to determine the final resources needed at each station by taking into account worker fatigue and shift rotation, using 1 reserve for each 5 front-line staff to allow for two 30-minute breaks and one 1-hour lunch break. For the actual field exercise with a duration 1.25 hour, the county used $5 \%$ of the calculated labor. In this case, the targeted treatment population is 450 households.

\subsubsection{Post event analysis and comparison of planned versus observed distribution}

It is noteworthy that using our staff allocation and assignments for the Anthrax field exercise, DeKalb achieved the highest throughput among all other counties that conducted the same scale of Anthrax exercise simultaneously at various locations, with the labor usage at or below the other counties. Specifically, DeKalb exceeded the targeted 450 households, and it processed 50\% more individuals compared to the second place county. None of the other counties achieved the targeted 450 households. The external evaluators commented that DeKalb produced the most efficient floor plan (with no path crossing), the most cost-effective dispensing (lowest labor/throughput value), and the smoothest operations (shortest average wait time, average queue length, equalized utilization rate). The study proves that even without historical data, using our system one can plan ahead and be able to wisely estimate the required labor resources.

During the field exercise, student volunteers from Georgia Institute of Technology and from Emory University were on hand taking measurements at various stations to collect firsthand data for post-event analysis. Some volunteer workers also walked through the entire process with individual clients to sample the cycle times.

Figure 6 shows the histograms of the active triage in the assembly point, passive triage in the POD, and the drug dispensing time, respectively. It also shows the associated fitted distribution versus the planned distribution for each of the three stations. 

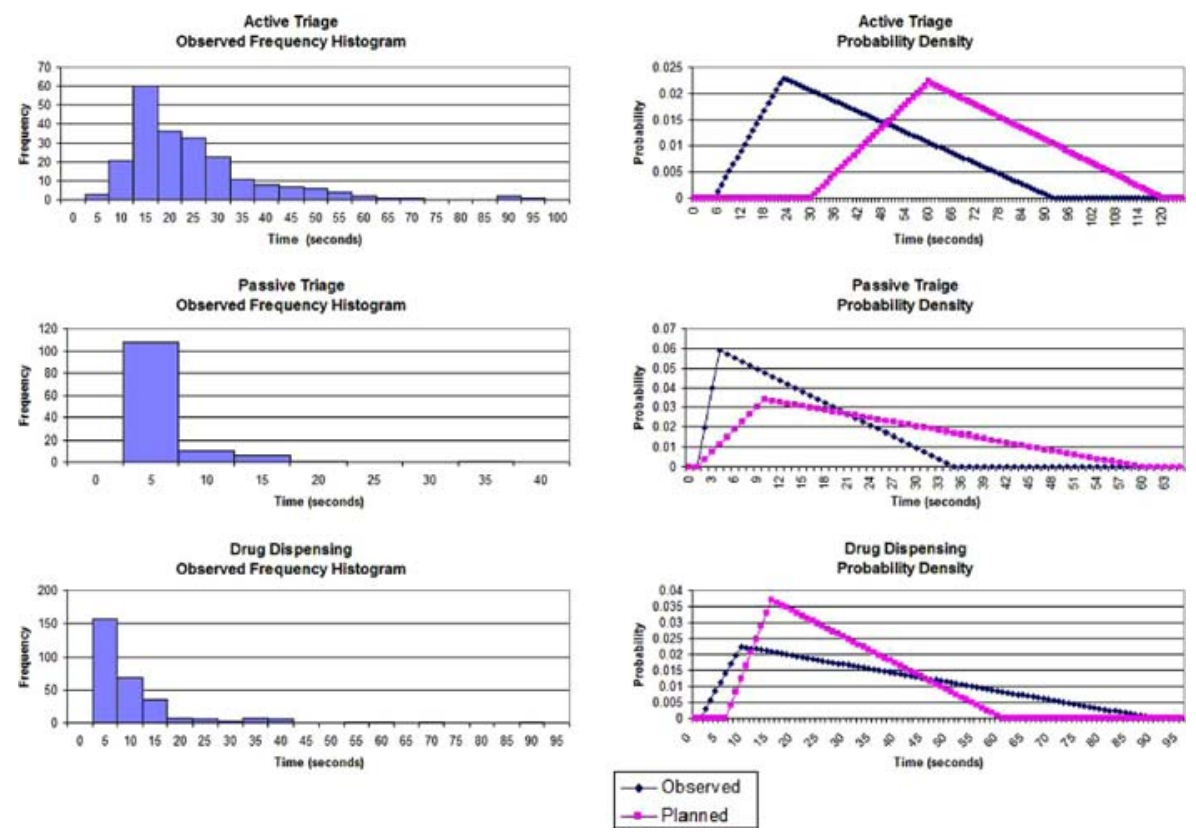

Fig. 6 Histograms for the Active Triage in the Assembly Point, Passive Triage in POD, and Drug Dispensing in POD, as collected during the actual anthrax exercise. For each station, the data is fitted and compared with the planned distribution

We remark that since there were fewer than 20 clients entering the Medical Evaluation and Mental Health Evaluation stations, the resulting estimated probability functions are deemed to be unreliable. Further, in real scenarios, due to educational broadcasts on television, newspaper and other mass media, and to announcements in community centers and public shelters, clients would normally be somewhat educated on the dispensing process and on issues related to existing medical conditions. Thus, it is expected that the flow for these two stations will remain relatively low. We also highlight the fact that the estimated dispensing time is indeed very close to the observed dispensing time.

A summary of the fitted probability distributions is shown in Table 3 below. Time units are in seconds.

Table 3 Anthrax dispensing study

\begin{tabular}{lll}
\hline & Distribution & (min, mean, max) seconds \\
\hline Observed distribution for & assembly point & simulation \\
Triage & Triangular & $(5,23,92)$ \\
Med Eval & Triangular & $(24,109,265)$ \\
Mental Health Eval & Triangular & $(51,73,101)$ \\
Observed distribution for point-of-dispensing simulation \\
Bus ride & Triangular & $(960,1388,1956)$ \\
Triage & Triangular & $(1,4,35)$ \\
Drug Dispensing & Triangular & $(1,9,90)$ \\
Med Eval & Triangular & $(26,93,256)$ \\
Mental Health Eval & Triangular & $(20,102,249)$ \\
\hline
\end{tabular}


Table 4 Staff allocation with the observed distributions

\begin{tabular}{|c|c|c|c|c|c|c|c|c|c|c|}
\hline \multirow[b]{2}{*}{$\begin{array}{l}\% \\
\text { Household }\end{array}$} & \multicolumn{4}{|c|}{ Assembly point } & \multicolumn{5}{|c|}{ Point-of-dispensing, POD } & \multirow[b]{2}{*}{ Total staff } \\
\hline & $\begin{array}{l}\text { Active } \\
\text { triage }\end{array}$ & Medical & $\begin{array}{l}\text { Mental } \\
\text { health }\end{array}$ & $\begin{array}{l}\text { Total } \\
\text { staff }\end{array}$ & $\begin{array}{l}\text { Passive } \\
\text { triage }\end{array}$ & Medical & $\begin{array}{l}\text { Mental } \\
\text { health }\end{array}$ & $\begin{array}{l}\text { Drug } \\
\text { dispensing }\end{array}$ & $\begin{array}{l}\text { Total } \\
\text { staff }\end{array}$ & \\
\hline 4 & 4 & 2 & 2 & 8 & 2 & 2 & 2 & 3 & 9 & 17 \\
\hline 8 & 7 & 2 & 2 & 11 & 3 & 2 & 2 & 6 & 13 & 24 \\
\hline 12 & 11 & 2 & 2 & 15 & 4 & 2 & 2 & 9 & 17 & 32 \\
\hline 16 & 14 & 2 & 2 & 18 & 5 & 2 & 2 & 11 & 20 & 38 \\
\hline 20 & 22 & 2 & 2 & 26 & 7 & 2 & 2 & 14 & 25 & 51 \\
\hline 40 & 45 & 2 & 2 & 49 & 13 & 2 & 2 & 28 & 45 & 94 \\
\hline 60 & 75 & 3 & 3 & 81 & 24 & 2 & 2 & 55 & 83 & 164 \\
\hline 80 & 76 & 4 & 3 & 83 & 25 & 3 & 2 & 58 & 88 & 171 \\
\hline 100 & 101 & 5 & 4 & 110 & 33 & 3 & 3 & 76 & 115 & 225 \\
\hline
\end{tabular}

We performed simulations using the observed distributions from the Anthrax exercise and contrasted these simulations with those obtained from the estimated distributions used for planning (as shown in Figs. 6(a)-(c) and Tables 2 and 3). The arrival process was assumed to be Poisson. The influx was controlled by (a) varying the mean of the probability distribution of the inter-arrival time (exponential), and (b) the number of people entering the system at each arrival. The appropriate average influx was determined by experimenting with different arrival parameter settings so as to achieve the required throughput.

Table 4 shows the staff allocation using the observed distributions. Figures 7(a) and (b) contrast the resource allocation results. Clearly, and as expected, the staff requirement for the "Planned" distribution exceeds that of the "Observed" distribution in every scenario. This can be explained by the fact that estimated distribution tends to be conservative to avoid underestimation of required resources needed in the actual exercise.

Below are some planning and logistics issues that were observed during the field exercise.

- The Active Triage stations at Assembly Points require more labor to man than was planned. Although compared to the planned distribution, the observed distribution indicates fewer staff assigned to man the Active Triage, in this case, the observed distribution is not reliable. Although it is based on over 300 observations, it does not reflect the actual triage properly. Indeed, during the exercise, many individuals went through active triage without staff intervening to address their concerns. This was due to a lack of available staff. Hence, in an actual event (or in a future exercise) more staff than planned for in this exercise will be needed.

- The use of large multi-language signs proved to be very helpful, overcoming the overwhelming delays and chaos due to language problems in the Arlington studies (Giovachino and McCue, 2003).

- The setup of Assembly Points in open parking lots appears to facilitate convenient and direct paths to various stations within the Assembly Point. However, some clients were overcome by heat, and this added to the burden of emergency response medical health personnel.

- The use of cards for labeling responsibility of different staff types is insufficient. Use of colored vests or T-shirts will be more appropriate.

- The bus route must be indicated more clearly; some bus drivers were lost in route between Assembly Point and POD, even though security officers were in place to direct traffic. This created major fluctuations in bus travel times, in spite of the fact that the traffic path had been cleared solely for the Anthrax exercise. 

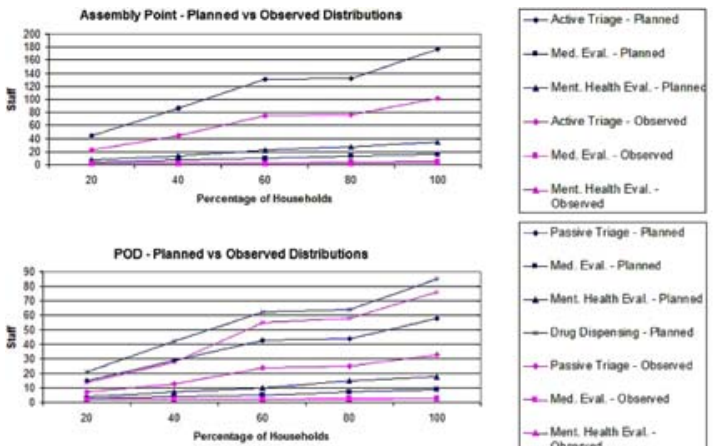

$\rightarrow$ Passive Triage - Planned

- Mad Eval - Plames

+Mant Heanh Eval - Plamid

-Orug Dispen ming - Planned

C.Passive Triage-Obsened

- Mad Eval-observes

- Ment Hedith Erat.

Observed

Orug Dispensing . Otserved
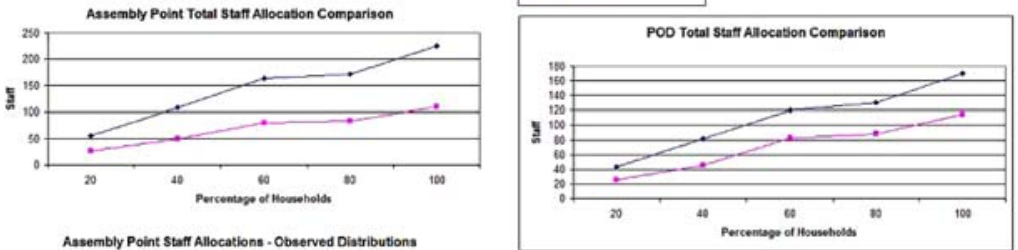

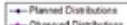

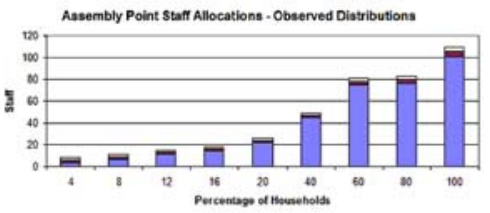

a Ment. Health Eval.

- Med. Eval.

$\square$ Active Triage
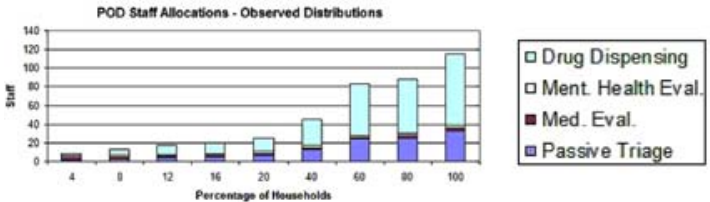

Fig. 7 (a) Comparison of staff allocations for a 12-hour shift at each station of an Assembly Point (Planned versus Observed distributions. (b) Comparison of staff allocations for a 12-hour shift at each station of a POD (Planned versus Observed distributions). (c) Comparison of total staff allocations for a 12-hour shift for an Assembly Point and a POD (Planned versus Observed distributions). (d) Resource allocation with observed distributions for a 12-hour shift for a single Assembly Point and POD

- The use of personnel on the bus while driving to the POD was very helpful in terms of informing clients. However, some clients became nervous during the ride, and it would therefore be appropriate to assign mental health counselors on the bus as well. There is a need for loudspeakers to ensure that everyone on the bus can hear the announcements.

- The use of a high-school gymnasium for drug dispensing proved to be a good choice, as its wide-open space allowed ease of queue formation and client flow and movement.

- Clients had to walk a rather long distance to reach the Passive Triage from the point where the buses dropped them off. This might create some confusion in a real situation. The Passive Triage should be close to the point where the clients are dropped off at POD, or greeting staff should be available to lead the clients into the Passive Triage stations.

- The design of the POD was a dramatic improvement over the Arlington field study since no paths within the POD were crossed, thus creating no extra confusion for clients as they moved within different stations in the POD.

- The location of restrooms was not taken into account when designing the POD. Within the POD, the restrooms were located between the POD entrance and Passive Triage. Thus, 
anyone (staff or client) going to the restroom had to walk against the client flow. Also, within the Assembly Point, no sign was posted as to where the restrooms were, causing confusion for some of the clients. Thus, signs must be prominently displayed, and the design of the POD layout with respect to the restroom locations must be considered carefully.

- The staff at the Mental Health Evaluation were performing at a slower pace than normal; perhaps they felt there was no urgency during the exercise. In a real situation, there maybe more people panicking and getting anxiety attacks. In fact, during the exercise, there were several individuals who actually thought that it was a real bio-attack, and they became rather nervous and agitated. In a real scenario, staff need to understand that they must handle these individuals efficiently.

- Clients should sign out by putting their numbers and time stamp on the sign-off sheet during the exit process, instead of searching for the number along the given sheet. The latter created a long queue during the exit process.

Although this was only a field exercise, elements of human anxiety-caused by not knowing in advance the full details of the process and steps within the Assembly Point and the POD areas-were apparent. Thus, the Medical and Mental Health Evaluation stations proved to be important, even though only a small percentage of observed clients needed such assistance. In an actual terror attack, it is likely that a major source of problems will not be due to the attack itself, but to the confusion and panic caused in the minds of people. Thus, psychological factors should be considered when preparing the dispensing plans. The planning simulation performed for the DeKalb anthrax exercise did not include psychological factors, but we expect these will be addressed in subsequent field exercises.

\subsubsection{Redesign of dispensing layout}

Based on the post event analysis and discussion among county officials, it was determined that the option of combining the Assembly Point and the POD into one facility should be explored. To understand the implications of such a redesign on resource usage and flow within the clinic, we performed a simulation study to facilitate a comparison analysis. Specifically, this study aims to compare the following:

- Personnel needed to staff the stations within a dispensing center, and the associated patient flow within the center;

- In the case of a catastrophic event (e.g., collapse of a POD due to targeted attack by bioterrorist, or to contamination via wide-spread infection due to contagious individuals) how well can we manage dispensing using the remaining operating PODs;

- Security personnel needed in each case.

For the discussion below, we will label the strategy of combining the Assembly Point and POD as Combined, and the original dispensing strategy (described in Sections 2.2.1 and 2.2.2) as Disjoint.

Figure 8 shows the Combined POD. It consists of four main blocks: Active Triage, Medical Evaluation, Mental Health Evaluation and Drug Dispensing. Patients first arrive at Triage from security. From the Triage, about $2 \%$ of the patients go to Medical Evaluation, $2 \%$ go to Mental Health Evaluation, $95 \%$ go to Drug Dispensing, and the remaining $1 \%$ exit the system.

Upon entering Medical Evaluation for the first time, about $50 \%$ of the patients go to Drug Dispensing, 35\% exit the system, and 15\% go to Mental Health Evaluation. Upon entering 


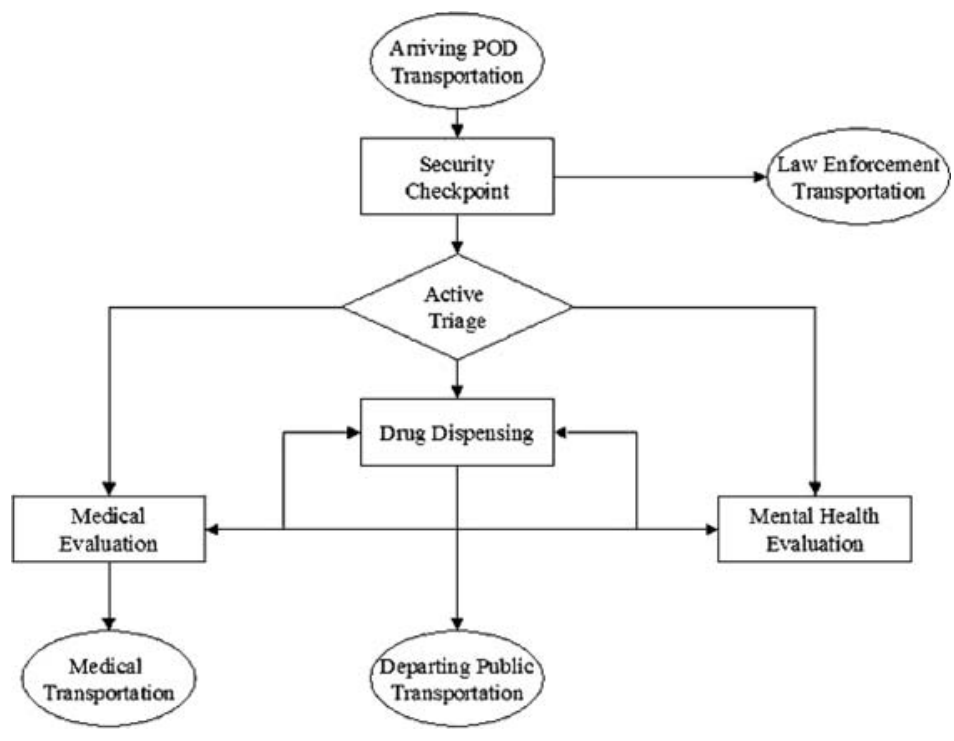

Fig. 8 Combined (New) POD Flow diagram

Mental Health Evaluation for the first time, 50\% go to Drug Dispensing, 35\% exit the system, and $15 \%$ go to Medical Evaluation.

When patients enter Medical Evaluation for the second time, $50 \%$ go to Drug Dispensing, and $50 \%$ exit the system. Similarly, when patients enter Mental Health Evaluation for the second time, $50 \%$ go to Drug Dispensing, and 50\% exit the system.

Figure 9(a) and Table 5 contrast the staff allocation to man various stations for a 12-hour shift for the Disjoint versus the Combined cases. The $x$-axis corresponds to the percentage of households processed out of the total 86,400 (100\%) targeted households for each POD in a 12-hour period. Contrasting the two dispensing strategies, as expected, fewer staff are assigned in every station for each of the throughput requirements for the Combined case. The

Table 5 Staff allocation with observed distribution for a single POD in 12-hour shift: Disjoint versus Combined

\begin{tabular}{|c|c|c|}
\hline & Disjoint & Combined \\
\hline$\%$ & $\begin{array}{ll}\text { Mental Drug } \quad \text { Total }\end{array}$ & Mental Drug \\
\hline
\end{tabular}

Household Triage Medical health dispensing staff Triage Medical health dispensing staff disjoint

\begin{tabular}{rrrrrrrrrrrr}
\hline 4 & 6 & 4 & 4 & 3 & 17 & 4 & 2 & 2 & 3 & 11 & 0.65 \\
8 & 10 & 4 & 4 & 6 & 24 & 7 & 2 & 2 & 6 & 17 & 0.71 \\
12 & 15 & 4 & 4 & 9 & 32 & 10 & 2 & 2 & 8 & 22 & 0.69 \\
16 & 19 & 4 & 4 & 11 & 38 & 14 & 2 & 2 & 11 & 29 & 0.76 \\
20 & 29 & 4 & 4 & 14 & 51 & 20 & 2 & 2 & 14 & 38 & 0.74 \\
40 & 58 & 4 & 4 & 28 & 94 & 40 & 2 & 2 & 27 & 71 & 0.75 \\
60 & 99 & 5 & 5 & 55 & 164 & 61 & 2 & 2 & 41 & 106 & 0.65 \\
80 & 101 & 7 & 5 & 58 & 171 & 72 & 2 & 2 & 54 & 130 & 0.76 \\
100 & 134 & 8 & 7 & 76 & 225 & 93 & 2 & 2 & 68 & 165 & 0.73 \\
\hline
\end{tabular}



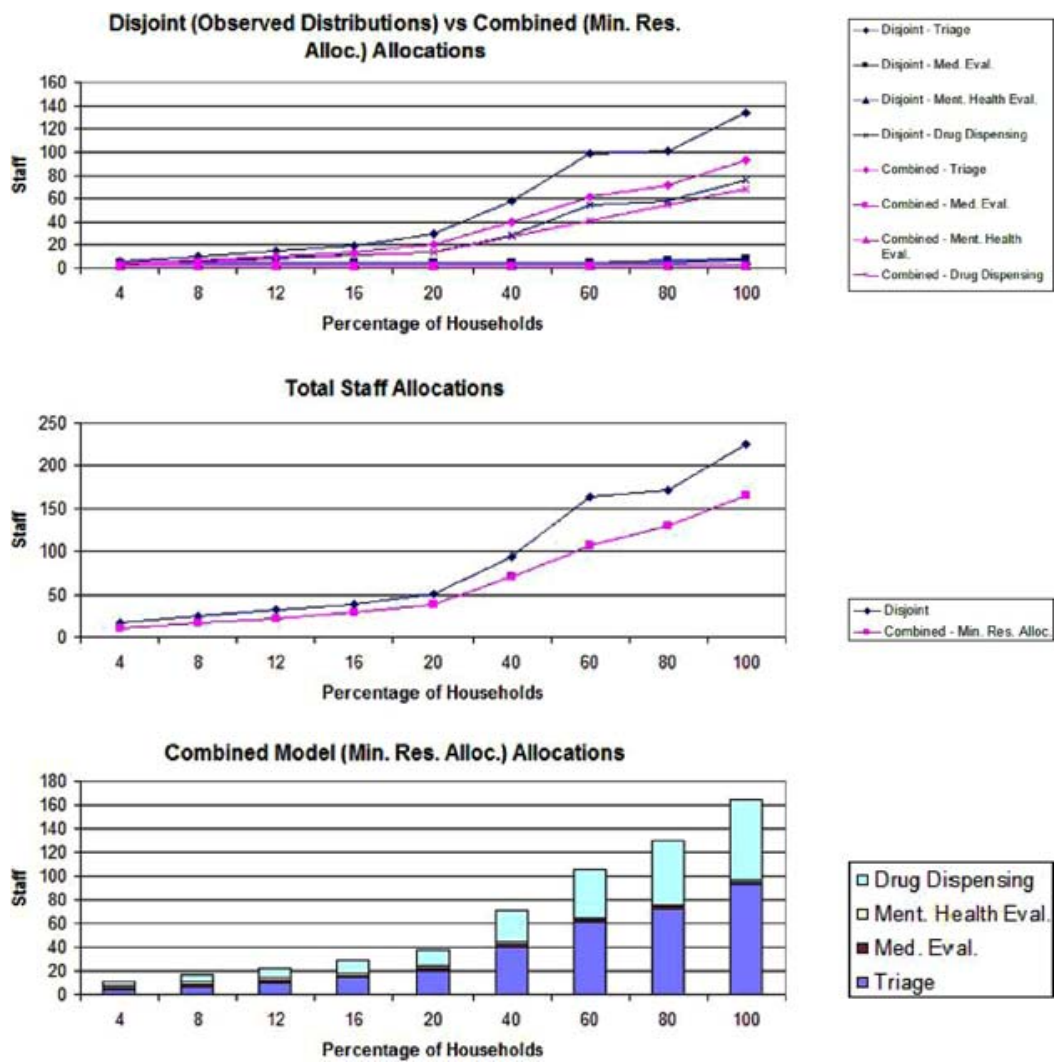

Fig. 9 (a) Comparison of staff allocation at each station for a 12-hour shift for Disjoint versus Combined strategies with objective of minimizing resource allocation while satisfying the required target number of households. (b) Comparison of total staff allocation for a 12-hour shift for Disjoint versus Combined strategies with objective of minimizing resource allocation while satisfying the required target number of households. (c) Resource allocation with observed distributions for a 12-hour shift for a single Combined POD

Combined model consistently uses between $65 \%$ to $76 \%$ of the assigned staff needed for the Disjoint model. Figure 9(b) contrasts the total staff usage.

From the barcharts in Fig. 9(c), we observe a below linear correlation between staff usage versus required throughput in the Combined case. Recall that a similar observation was made earlier in the Disjoint case. In both the Disjoint and Combined cases, the utilization rate at most stations is above $80 \%$ capacity. Average cycle times and associated half-widths are shown in Table 6 (the first four columns); the times are within the range of $42 \mathrm{~min}$ to $130 \mathrm{~min}$.

One of the issues that the state emergency preparedness team would like to explore is the ability for the Combined POD to increase throughput level, beyond the 86,400 households per 12-hour shift period. One method of gauging this involves using the minimum staff resources required for the Disjoint case to service 86,400 households, and applying these same resources to the Combined POD with the objective of maximizing the overall throughput. Figure 10(a) and Table 7 show the potential improvement. Using the same staff resources, the Combined POD consistently increases throughout by nearly two to three fold for each of the original target levels. With this increased throughput, the estimated time for dispensing to the entire regional households of 864,000 can be accomplished within 8-12 hours by 5 Combined 
Table 6 Average Cycle Times (in seconds)_-Disjoint versus Combined

\begin{tabular}{|c|c|c|c|c|c|c|}
\hline \multirow{3}{*}{$\begin{array}{l}\% \\
\text { Household }\end{array}$} & & & \multicolumn{4}{|c|}{ Combined } \\
\hline & \multicolumn{2}{|c|}{$\begin{array}{c}\text { Disjoint } \\
\text { Min resource }\end{array}$} & \multicolumn{2}{|c|}{ Min resource } & \multicolumn{2}{|c|}{$\begin{array}{l}\text { Maximize throughput } \\
\text { use same resource as Disjoint }\end{array}$} \\
\hline & Average & Half width & Average & Half width & Average & Half width \\
\hline 20 & 8055.52 & 84.57 & 7124.49 & 77.75 & 7942.26 & 202.75 \\
\hline 40 & 7823.87 & 70.46 & 7655.52 & 48.44 & 8489.11 & 165.02 \\
\hline 60 & 3182.18 & 41.95 & 7404.35 & 43.76 & 9194.67 & 138.29 \\
\hline 80 & 2571.18 & 50.26 & 2831.21 & 52.47 & 5589.50 & 121.87 \\
\hline 100 & 2829.03 & 41.77 & 3827.08 & 40.29 & 2736.08 & 117.97 \\
\hline
\end{tabular}
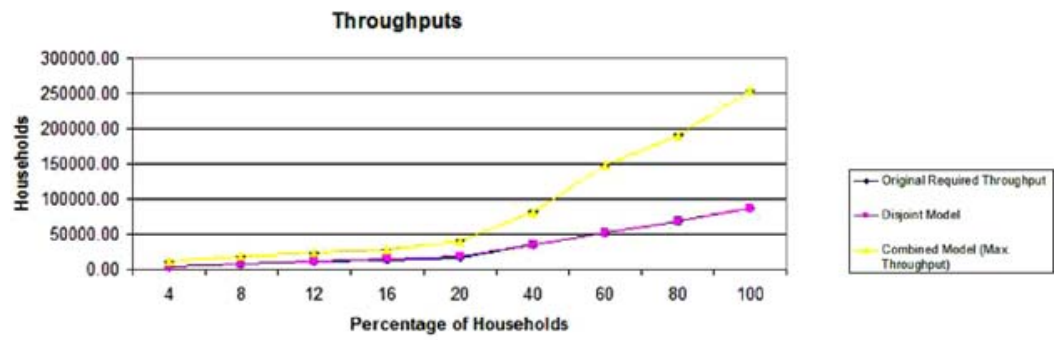

Disjoint (Observed Distributions) vs Combined (Max. Through puts) Allocations
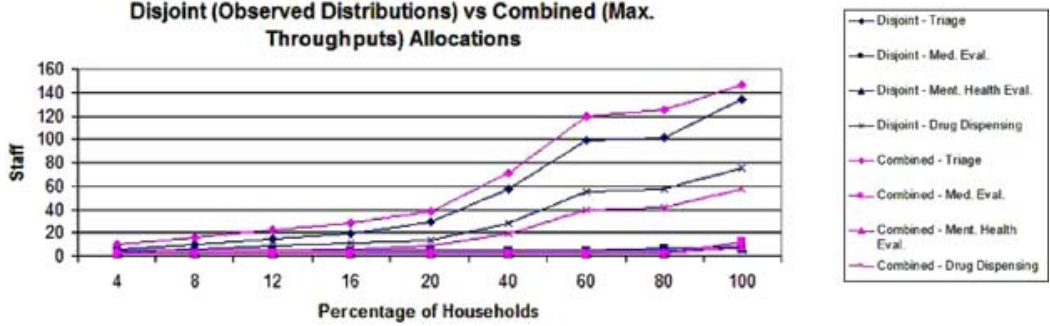

Fig. 10 (a) Comparison of throughput for Disjoint versus Combined strategies when same staff resources are used for a 12-hour shift. The resources are obtained by minimizing resource allocation while satisfying the required target number of households for the Disjoint model. This staff resource level is then applied to the Combined case in which maximum throughput is sought. (b) Comparison of Staff allocation at each station for Disjoint versus Combined strategies when same staff resources are used for a 12-hour shift. The resources are obtained by minimizing resource allocation while satisfying the required target number of households for the Disjoint model. This resource level is then applied to the Combined case in which maximum throughput is sought

PODs (versus 24 hours by 5 Disjoint setups), or 10-15 hours when one POD collapses as a result of catastrophic shutdown.

Contrasting Tables 6 and 7, we observe that as many more clients are pumped into the system in the Combined maximize-throughput scenario, the average cycle time spent by an individual lengthens in all except one scenario (contrast Table 6, columns 4 and 6). We can compensate by enforcing a tight cycle-time constraint, which will result in a slight decrease in the final throughput. The results reported here are aimed at illustrating how far one can 
Table 7 Maximize throughput for each POD within a 12-hour shift Disjoint versus Combined model, when same resources are used

\begin{tabular}{|c|c|c|c|c|c|c|}
\hline \multirow{2}{*}{$\begin{array}{l}\% \\
\text { Household }\end{array}$} & \multirow{2}{*}{$\begin{array}{l}\text { Original required } \\
\text { throughput }\end{array}$} & \multicolumn{2}{|c|}{$\begin{array}{c}\text { Disjoint } \\
\text { model }\end{array}$} & \multicolumn{2}{|c|}{$\begin{array}{c}\text { Combined } \\
\text { Maximize throughput }\end{array}$} & \multirow{2}{*}{$\begin{array}{l}\text { Combined } \\
\text { to disjoint }\end{array}$} \\
\hline & & Average & Half width & Average & Half width & \\
\hline 4 & 3456.00 & 3603.90 & 9.55 & 10369.40 & 0.95 & 2.88 \\
\hline 8 & 6912.00 & 7117.40 & 16.08 & 18427.50 & 730.92 & 2.59 \\
\hline 12 & 10368.00 & 10860.20 & 7.26 & 23206.90 & 831.87 & 2.14 \\
\hline 16 & 13824.00 & 14058.95 & 32.79 & 27738.70 & 737.21 & 1.97 \\
\hline 20 & 17280.00 & 17906.05 & 19.28 & 41258.30 & 979.74 & 2.30 \\
\hline 40 & 34560.00 & 34598.15 & 40.09 & 82171.90 & 1030.56 & 2.38 \\
\hline 60 & 51840.00 & 52011.55 & 58.17 & 148610.00 & 41.74 & 2.86 \\
\hline 80 & 69120.00 & 69192.90 & 60.84 & 191258.25 & 1384.95 & 2.76 \\
\hline 100 & 86400.00 & 86925.25 & 56.71 & 253450.75 & 61.36 & 2.92 \\
\hline
\end{tabular}

Table 8 Staff allocation within each station in the dispensing center where throughput is maximized for each POD within a 12-hour shift. Disjoint versus Combined model, when same resources are used

\begin{tabular}{|c|c|c|c|c|c|c|c|c|c|}
\hline \multirow[b]{2}{*}{$\begin{array}{l}\% \\
\text { Household }\end{array}$} & \multirow[b]{2}{*}{$\begin{array}{l}\text { Total } \\
\text { staff }\end{array}$} & \multicolumn{4}{|c|}{ Disjoint } & \multicolumn{4}{|c|}{ Combined } \\
\hline & & Triage & Medical & $\begin{array}{l}\text { Mental } \\
\text { health }\end{array}$ & $\begin{array}{l}\text { Drug } \\
\text { dispensing }\end{array}$ & Triage & Medical & $\begin{array}{l}\text { Mental } \\
\text { health }\end{array}$ & $\begin{array}{l}\text { Drug } \\
\text { dispensing }\end{array}$ \\
\hline 4 & 17 & 6 & 4 & 4 & 3 & 10 & 2 & 2 & 3 \\
\hline 8 & 24 & 10 & 4 & 4 & 6 & 16 & 2 & 2 & 4 \\
\hline 12 & 32 & 15 & 4 & 4 & 9 & 23 & 2 & 2 & 5 \\
\hline 16 & 38 & 19 & 4 & 4 & 11 & 28 & 2 & 2 & 6 \\
\hline 20 & 51 & 29 & 4 & 4 & 14 & 38 & 2 & 2 & 9 \\
\hline 40 & 94 & 58 & 4 & 4 & 28 & 71 & 2 & 2 & 19 \\
\hline 60 & 164 & 99 & 5 & 5 & 55 & 120 & 2 & 2 & 40 \\
\hline 80 & 171 & 101 & 7 & 5 & 58 & 125 & 2 & 2 & 42 \\
\hline 100 & 225 & 134 & 8 & 7 & 76 & 147 & 12 & 8 & 58 \\
\hline
\end{tabular}

push the throughput in the Combined case, when the average cycle time is roughly within the range of $150 \mathrm{~min}$.

Figure 10(b) and Table 8 contrast staff usage in each of the tasks for the Disjoint versus the Combined scenarios when the same number of staff is provided.

The results reported herein are only a first-order analysis; extensive studies are underway to simulate various scenarios involving different unexpected events occurring within the Disjoint and the Combined cases to better understand tactics and strategies that one should employ.

We remark that in both cases, a security check is performed before each patient arrives at Triage. Security is generally performed by police, although in the case of a biological attack, it could be performed by military personnel. It is accepted that the security resources needed prior to Triage and those needed within the dispensing process are similar in both the Disjoint and Combined strategies. However, in the Disjoint case, additional security services are required to man/secure the bus and to direct the bus while traveling to and from the POD. Thus, it is expected that overall, more police / military personnel will be required for the Disjoint strategy. 
As a tradeoff, the Disjoint strategy seems to offer a more secure way to a handle a catastrophic event. If an Assembly Point is rendered inoperable (e.g., because of an attack or contamination), this will affect the individuals locally, but will not affect dispensing in associated PODs, except that the inflow of individuals will cease from the inoperable Assembly Point. Good communication and planning will be required to ensure that individuals and buses traveling to the affected Assembly Point will be re-directed to other Assembly Points. Staff adjustments will be needed to increase personnel in the remaining operable Assembly Points, or to set up an alternative Assembly Point at another location. In the case that a POD collapses, either an alternative POD can be established, or additional personnel can be deployed at the remaining operable PODs to ensure that the cumulative dispensing rate of all operable PODs is maintained. However, assuming a thorough security and medical check is performed at Assembly Points, then for the Disjoint strategy, the likelihood of a POD collapse is somewhat remote, since individuals arriving at PODs will have already been thoroughly screened at the Assmembly point. This applies to a collapse due to either a terrorist incident or to contamination through infectious disease, as suspect/infectious individuals will not be allowed to proceed to a POD.

In the Combined strategy, Security and first-time Active Triage are in close vicinity of the dispensing station. A security or contamination incident will thus affect the dispensing process directly, and a POD shutdown will influence the overall dispensing rate of the entire region. In this case, a backup POD in a separate location can be established, or un-affected healthcare workers can be re-assigned to existing PODs to increase processing capacity so as to maintain the overall dispensing rate.

The type of disaster being confronted (e.g., Anthrax, Plague, Tularemia, Smallpox, or Botulinum) will likely play a role in the choice of dispensing strategy (Disjoint or Combined). Depending on the strategy selected, backup plans will be different, and the level of security and military personnel, as well as the number of healthcare workers required, will vary. The key point is that flexibility - both of staff to be able to perform a number of different duties, and of facility to be readily reconfigured on-the-fly-is needed to respond to dynamic changes. We expect that a feasible approach is to set up a dispensing center based on the best estimates and analysis available, and then be prepared to reconfigure it while it operates. Clearly, our decision support system, which facilitates analysis of "what-if" scenarios, will serve as an invaluable tool for planning such reconfigurations.

\section{Discussion}

This paper describes a standalone large-scale decision support system, $\mathrm{RealOpt}^{\odot}$, that couples a flexible simulation environment with fast powerful heuristic algorithms that are tailored for resource allocation and emergency response planning. Using this system, the computational time required to complete all tests for the CDC smallpox scenarios was less than a single CPU minute, as opposed to five to ten hours (for a feasible solution) on a commercial simulation system (Mason and Washington, 2003). The system's fast computational time enables its use in large-scale studies, like the Anthrax planning exercise discussed herein involving a region with 864,000 households. The combined computational effort required for this exercise was roughly $30 \mathrm{~min}$ for all scenarios considered, demonstrating that $\mathrm{RealOpt}^{\odot}$ offers a very promising avenue for pursuing a comprehensive investigation involving a more diverse set of scenarios, and justifying work towards development of a robust system that can be widely deployed for use by state, local, and tribal health practitioners. 
It is noteworthy that using our staff allocation and assignments for the Anthrax field exercise, DeKalb county achieved the highest throughput among all counties that simultaneously conducted the same scale of Anthrax exercise at various locations, with labor usage at or below the other counties. Indeed, DeKalb exceeded the targeted 450 households, and it processed $50 \%$ more individuals compared to the second place county. None of the other counties achieved the targeted 450 households. The external evaluators commented that DeKalb produced the most efficient floor plan (with no path crossing), the most cost-effective dispensing (lowest labor/throughput value), and the smoothest operations (shortest average wait time, average queue length, equalized utilization rate). The study proves that even without historical data, using our system one can plan ahead and be able to wisely estimate the required labor resources.

The exercise also revealed many areas that need attention during the operations planning and design of dispensing centers. The type of disaster being confronted (e.g., biological attack, infectious disease outbreak, or a natural disaster) also dictates different design considerations with respect to the dispensing clinic, facility locations, dispensing and backup strategies, and level of security protection. Depending on the situation, backup plans will be different, and the level of security and military personnel, as well as the number of healthcare workers required, will vary. The key point is that flexibility is needed to respond to dynamic changes. A feasible approach is to set up a dispensing center based on the best estimates and analysis available, and then be prepared to reconfigure it while it operates. Our decision support system, which facilitates analysis of "what-if" scenarios, will serve as an invaluable tool for planning and reconfigurations.

This study confirms that: (1) A real-time decision support system is viable through careful design of a standalone simulator coupled with powerful tailor-designed optimization solvers. (2) The system can overcome the computational bottleneck observed when using existing commercial systems, as observed by the CDC reports. (3) The flexibility of performing empirical tests quickly means the system is amenable for use in training and preparation, and for strategic planning before and during an emergency situation. It also allows for "virtual field exercises" to be performed on the decision support system, offering insight into operations flow and bottlenecks when mass dispensing occurs to a region with significant large population. (4) It is possible to carefully design empirical experiments and catastrophic scenarios so as to reveal insights regarding emergency center operations. (5) The modular design and flexible implementation enables future expansion and modification regarding emergency center design with respect to treatment for different biological threats or disease outbreaks.

Working with various state and county emergency response departments, numerous additional empirical tests will be performed to fine-tune the system, and modules will be developed to address different biological attacks and infectious disease outbreaks. The system is freely available for use by state, local, and tribal public health departments for emergency planning.

\section{References}

Alibek, K. (1999). Biohazard: The Chilling True Story of the Largest Covert Biological Weapons Program in the World-Told from Inside by the Man Who Ran It. Random House, New York (N.Y.).

Altiok, T. (1997). Performance Analysis of Manufacturing Systems. Springer-Verlag, New York.

Barnett, D.J., R.D. Balicer, D. Blodgett, A.L. Fews, C.L. Parker, and J.M. Links. (2005). "The Application of the Haddon Matrix to Public Health Readiness and Response Planning." Environmental Health Perspectives, 113(5), 561-566.

Bellamy, R.J. and A.R. Freedman. (2001). "Bioterrorism.” QJM, 94(4), 227-334. 
Bravata, D.M., K.M. McDonald, W.M. Smith, C. Rydzak, H. Szeto, D.L. Buckeridge, C. Haberland, and D.K. Owens. (2004). "Systematic Review: Surveillance Systems for Early Detection of Bioterrorism-Related Diseases." Annals of Internal Medicine, 140(11), 910-922.

Buzacott J.A. and J.G. Shanthikumar. (1993). Stochastic Models of Manufacturing Systems. Prentice Hall, New Jersey.

Centers for Disease Control. (2002 August). "Smallpox Vaccination Clinic Guide: Logistical Considerations and Guidance for State and Local Planning for Emergency Large-Scale, Post-Event, Voluntary Administration of Smallpox Vaccine."

Centers for Disease Control and Prevention. (2002 November). Maxi-Vac 1.0, Beta Test Version.

Centers for Disease Control and Prevention. (2002). "Evaluation of Post Exposure Antibiotic Prophylaxis to Prevent Anthrax." Morbidity and Mortality Weekly Report, 51(03).

Clizbe, J.A. (2004). "Challenges in Managing Volunteers During Bioterrorism Response." Biosecurity and Bioterrorism: Biodefense Strategy, Practice, and Science, 2(4), 294-300.

Dallery, Y. and Y. Frein. (1993). "On Decomposition Methods for Tandem Queueing Networks with Blocking." Oper. Res., 41(2), 386-399.

Drenkard, K., G. Rigotti, D. Hanfling, T.L. Fahlgren, and G. LaFrancois. (2002). "Healthcare System Disaster Preparedness, Part 1: Readiness Planning.” Journal of Nursing Administration, 32(9), 461-469.

Eglese, R.W. (1990). “Simulated Annealing: A Tool for Operational Research.” European J. Oper. Res., 46(3), 271-283.

Epstein, J., D.A.T. Cummings, S. Chakravarty, R. Singa, and D.S. Burke. (2002). "Toward a Containment Strategy for Smallpox Bioterror: An Individual-Based Computational Aapproach [working paper no. 31]." Brookings Institution, Johns Hopkins University Center on Social and Economic Dynamics.

Eubank, S. (2002). "Scalable, Efficient Epidemiological Simulation." In Proceedings of the 2002 ACM Symposium on Applied Computing (Madrid, Spain, March 11-14, 2002). SAC '02. ACM Press, New York, NY, 139-145. DOI=http://doi.acm.org/10.1145/508791.508819.

Fast, J.D., B.L. O’Steen, and R.P. Addis. (1995). “Advanced Atmospheric Modeling for Emergency Response.” Journal of Applied Meteorology, 34(3), 626-649.

Fauci, A.S. (2002). "Smallpox Vaccination Policy-The Need for Dialogue." N. Engl. J. Med., 346(17), 1319-1320.

Franz, D.R., P.B. Jahrling, A.M. Friedlander, D.J. McClain, D.L. Hoover, W.R. Bryne, J.A. Pavlin, G.W. Christopher, and E.M. Eitzen Jr. (1997). "Clinical Recognition and Management of Patients Exposed to Biological Warfare Agents.” JAMA, 278(5), 399-411.

Gani, R. and S. Leach. (2001). "Transmission Potential of Smallpox in Contemporary Populations." Nature, 414(13).

Gershwin, S.B. (1987). "An Efficient Decomposition Method for the Approximate Evaluation of Tandem Queues with Finite Storage Space and Blocking." Oper. Res., 35(2), 291-305.

Giovachino, M.J. and B.G. McCue. (2003 April). "Mini-Vac Model Documentation." Institute for Public Research, (IPR)10889.

Gross, D. and C.M. Harris. (1998). Fundamentals of Queueing Theory. Wiley Interscience.

Heavey, C., H.T. Papadopoulos, and J. Browne. (1993). "The Throughput Rate of Multistation Unreliable Production Lines." European J. Oper. Res., 68(1), 68-89.

Hopp, W.J. and M.L. Spearman. (2000). Factory Physics. McGraw-Hill/Irwin, New York (NY).

Hupert, N., G.M.L. Bearman, A.I. Mushlin, and M.A. Callahan. (2003). "Accuracy of Screening for Inhalational Anthrax after a Bioterrorist Aattack." Ann. Int. Med., 139(5), 337-345.

Hupert, N., A.J. Mushlin, and M.A. Callahan. (2002). "Modeling the Public Health Response to Bioterrorism: Using Discrete Event Simulation to Design Antibiotic Distribution Centers." Medical Decision Making, 22 (Suppl), S17-S25.

Kaplan, E.H. (2004). "Preventing Second-Generation Infections in a Smallpox Bioterror Attack." Epidemiology, 15(3), 264-270.

Kaplan, E.H., D.L. Craft, and L.M. Wein. (2002). "Emergency Response to a Smallpox Attack: The Case for Mass Vaccination." Proceedings of the National Academy of Sciences, 99(16).

Kaplan, E.H., D.L. Craft, and L.M. Wein. (2003). "Analyzing Bioterror Response Logistics: The Case of Smallpox." Mathematical Biosciences, 185(1), 33-72.

Kaufmann, A.F., M.T. Meltzer, and G.P. Schmid. (1997). “The Economic Impact of a Bioterrorist Attack: Are Prevention and Postattack Intervention Programs Justifiable?” Emerg. Infect. Dis., 3(2), 83-94.

Keim, M. and A.F. Kaufman. (1999). "Principles for Emergency Response to Bioterrorism." Ann. Emerg. Med., 34(2), 177-182.

Laguna, M. and R. Marti. (2003). Scatter Search: Methodology and Implementations in C. Kluwer Academic Publishers, Boston (MA). 
Larkin, G.L. and J. Arnold. (2004). "Ethical Considerations in Emergency Planning, Preparedness, and Response to Acts of Terrorism." Prehospital and Disaster Medicine, 18(3).

Larson, R.C. (2004). "Decision Models for Emergency Response Planning." In D. Kamien (ed.), The McGrawHill Handbook of Homeland Security. McGraw-Hill.

Law, A.M. and W.D. Kelton. (1991). Simulation Modeling and Analysis. McGrawhill, New York (NY).

Lee, E.K. and S. Maheshwary. (2004). Systems and Methods for Emergency Treatment Response and RealTime Staff Allocation for Bioterrorism and Infectious Disease Outbreak [copyright 2004, provisional patent June 2004, 2005]. Georgia Institute of Technology.

Lee, E.K., S. Maheshwary, and J. Mason. (2005). "Real-Time Staff Allocation for Emergency Treatment Response of Biological Threats and Infectious Disease Outbreak." Medical Decision Making. Competitive selected as INFORMS William Pierskalla Best Paper Award on research excellence in HealthCare and Management Science, November 2005.

Mason, J. and M. Washington. (2003). “Optimizing Staff Allocation in Large-Scale Dispensing Centers.” CDC report.

Meltzer, M., I. Damon, J.W. LeDunc, and D.J. Millar. (2001). "Modeling Potential Responses to Smallpox as a Bioterrorist Weapon.” Emerg. Infect. Dis., 7(6), 959-969

Nemhauser, G.L. and L.A. Wolsey. (1988). Integer and Combinatorial Optimization. Wiley, New York.

Nguyen, S., J.M. Rosen, and C.E. Koop. (2005). "Medicine Meets Virtual Reality 13: The Magical Next Becomes the Medical Now." Emerging Technologies for Bioweapons Defense, Amsterdam, IOS Press, The Netherlands Vol. 111, 356-361.

Noji, E.K. (2003). "Medical Preparedness and Response to Terrorism with Biological and Chemical AgentsPresent Status in USA.” International Journal of Disaster Medicine, 1(1), 51-55.

Okumura T., K. Suzuki, A. Fukuda, A. Kohama, N. Takasu, S. Ishimatsu, and S. Hinohara. (1998). "The Tokyo Subway Sarin Attack: Disaster Management, Part 1: Community Emergency Response.” Academic Emergency Medicine, 5, 613-617.

Papadopoulos, H.T., C. Heavey, and J. Browne. (1993). Queueing Theory in Manufacturing Systems Analysis and Design. Chapman and Hall, London.

RealOpt User Manual, Georgia Institute of Technology, 2004-2006.

Reis, B.Y. and K.D. Mandl. (2003). "Time Series Modeling for Syndromic Surveillance.” BMC Medical Informatics and Decision Making, 3(2).

Ross, S.M. (1995). Stochastic Processes. John Wiley and Sons.

Rotz, L.D. and J.M. Hughes. (2004). "Advances in Detecting and Responding to Threats from Bioterrorism and Emerging Infectious Disease.” Nature Medicine, 10(12 Suppl), S130-S136.

Scott, S. and C. Duncan. (2001). Biology of Plagues: Evidence from Historical Populations. Cambridge University Press, Cambridge.

Sidel, V.W., R.M. Gould, and H.W. Cohen. (2002). "Bioterrorism Preparedness: Cooptation of Public Health." Medicine \& Global Survival, 7(2), 82-89.

Spinellis, D. and C.T. Papadopoulos. (2000a). "A Simulated Annealing Approach for Buffer Allocation in Reliable Production Lines." Ann. of Oper. Res., 93(1-4), 373-384.

Spinellis, D. and C. Papadopoulos. (2000b). "Stochastic Algorithms for Buffer Allocation in Reliable Production Lines." Mathematical Problems in Engineering, 5(6), 441-458.

Tompkins, G. and F. Azadivar. (1995). "Genetic Algorithms in Optimizing Simulated Systems.” In Proceedings of the 27th Conference on Winter Simulation, Arlington (VA), ACM Press, New York (NY), 757-762.

Viswanadham, N. and Y. Narahari. (1992). Performance Modeling of Automated Manufacturing Systems. Prentice Hall, New Jersey.

Wein, L.M., D.L. Craft, and E.H. Kaplan. (2003). “Emergency Response to an Anthrax Attack.” In Proceedings of the National Academy of Sciences, 100(7), 4346-4351. 